\title{
Lignocellulose and White-Rot Basidiomycetes: Some Strategies for Their Potential Utilization
}

\author{
Vladimir Elisashvili E๐ George Kvesitadze
}

Durmishidze Institute of Biochemistry and Biotechnology, 0159 Tbilisi, Georgia

Lignocellulose composes more than $60 \%$ of plant biomass produced on earth. In addition, municipal and agro-industrial waste generation is fast increasing and causing pollution and many other environmental problems. The agro-industrial waste is a potentially vast source of energy, substrate for mushroom productions, biofertilizers, animal feed, enzymes, and other biochemical products. The most efficient decomposers of lignocellulose are the white-rot fungi because of the capability of these fungi to synthesize the relevant hydrolytic (cellulases and hemicellulases) and oxidative (ligninolytic) extracellular enzymes required to degrade the major components of the substrate-i.e., cellulose, hemicellulose, and lignin-into low-molecular-weight compounds that can be assimilated in fungi nutrition.

Recently, extensive research on these fungi has been conducted with the aim of isolating new organisms with tremendous secretion of ligninolytic enzymes as well as enzymes with properties important for their industrial application in the bioremediation of polluted, hazardous xenobiotic soils and industrial waters; biobleaching and biopulping; the textile, dye, and food industries; biotransformation of pharmaceutical and other intermediates; biosensor construction; cosmetics; medicine; analytic biochemistry. All these biotechnological applications require huge amounts of enzymes. However, their application is hindered because the enzymes that are presently being investigated are still expensive because of the low yield and high cost in production and isolation. Overexpression of laccase and peroxi- dases in heterologous systems has not been achieved yet, and they still have to be obtained from natural sources. Therefore, the search for powerful producers of enzymes and the development of alternative technologies for enzyme production are mandatory. Consequently, an overview of various approaches will be presented aiming to stimulate the production and yield of target enzymes as well as the analysis of the studies proving that the regulation of lignocellulolytic enzymes synthesis appears to be subject to the complex effects of nutritional, environmental, temporal, and genetic factors. Understanding of physiological mechanisms regulating enzyme synthesis in lignocellulose bioconversion could be useful for improving the technological process of edible and medicinal mushroom production.

Special attention will be paid to edible and medicinal mushrooms of the genus Pleurotus and Lentinus edodes (Berk.) Singer, which serve as the basis for various biotechnological and environmental applications. Some of these applications are traditional and are practiced throughout the world, and some need further research and development. Cumulative evidence indicates that the selection of an appropriate cultivation technique and plant residue adequate for fungus growth and target enzyme synthesis plays an important role in the development of an efficient biotechnology. Cultivation of Pleurotus strains and L. edodes on wheat straw, tree leaves, and cotton wastes as growth substrates showed clear physiological and biochemical changes taking place during plant raw material colonization and 
fructification. The vegetative growth of these edible mushrooms is characterized by the comparatively low activity of polysaccharases and high activity of ligninolytic enzymes. During the period of fruiting body formation, when mushroom growth demands a large quantity of plastic materials and energy, the secretion of cellulases and xylanase sharply increases to accelerate polysaccharide hydrolysis. In contrast, ligninolytic enzyme production appears to be, to some extent, repressed in preventing the expenses of constructive materials and energy.

In addition, the investigation of the hydrolytic and oxidative enzyme production during Pleurotus spp. and $L$. edodes cultivation is mandatory, because after harvesting the fruit bodies the residual spent substrate may become a cheap source of lignocellulolytic enzymes for several biotechnological applications, and it may be used as a bioremediation agent. 